\title{
Comparison of efficacy of chemical peeling with $25 \%$ trichloroacetic acid and $0.1 \%$ retinoic acid for facial rejuvenation
}

\author{
Selda Yildirim ${ }^{1}$, Mehmet Salih Gurel ${ }^{2}$, Sule Gungor ${ }^{3}$, Omur Tekeli $^{4}$, Dilek Canat ${ }^{5}$ \\ 'Dermatology Department, Atakent Acibadem Hospital, Acibadem Unıversity, Istanbul, Turkey \\ ${ }^{2}$ Dermatology Department, Istanbul Training and Research Hospital, Istanbul, Turkey \\ ${ }^{3}$ Dermatology Department, Okmeydanı Training and Research Hospital, Istanbul, Turkey \\ ${ }^{4}$ Dermatology Department, Omur Tekeli Clınıc, Istanbul, Turkey \\ ${ }^{5}$ Dermatology Department, Esenyurt State Hospital, Istanbul, Turkey
}

Adv Dermatol Allergol 2016; XXXIII (3): 199-205

DOI: 10.5114/ada.2016.60612

\begin{abstract}
Introduction: Skin aging is a problem which negatively affects the psyche of the person, social relations, as well as work life and health and which compels the patients to find appropriate treatment methods. Numerous treatment methods have been developed in order to delay aging and to reduce the aging effects in addition to having a younger, healthier and more beautiful facial appearance.

Aim: To compare the efficiency, cosmetic results and possible adverse effects of the peeling treatment with $25 \%$ trichloroacetic acid (TCA) and $0.1 \%$ retinoic acid for facial rejuvenation in patients presenting with skin aging. Material and methods: Fifty female patients in total presenting with medium and advanced degree skin aging were subject to this study. Two separate treatment groups were formed; the first group underwent chemical skin treatment with $25 \%$ TCA while the other group was applied with $0.1 \%$ retinoic acid treatment. Following the 4 months' treatment the patients were controlled three times in total for post lesional hypopigmentation, hyperpigmentation, scars, skin irritation and other possible changes per month. The pretreatment and first follow-up visit, and final control images were comparatively evaluated by three observers via specific software.

Results: The healing rates of the group subject to retinoic acid were statistically higher $(p<0.05)$ compared to patients in the TCA group in the final follow-up visit following the treatment according to the first and second observers. On the other hand, according to the third observer, patients applied with retinoic acid presented with higher healing rates compared to those treated with TCA, however; this rate was not statistically significant $(p>0.05)$. The frequency of TCA- and retinoic acid-associated adverse effects was similar in both groups $(p>0.05)$. As a result of both treatments, a reduction in the quality of life scores as well as a pronounced recovery $(p=0.001)$ in the quality of life of those patients with skin aging was observed.

Conclusions: The photo aging treatment option with $0.1 \%$ retinoic acid is cheaper and more feasible for patients compared to $25 \%$ TCA, and it is also as reliable and effective as TCA.
\end{abstract}

Key words: skin aging, photo aging, trichloroacetic acid, retinoic acid.

\section{Introduction}

Photo aging is a term used to describe the clinical and dermatopathological changes in the middle-aged and older adults chronically exposed to sunlight. It is clinically characterized by skin coarsening, wrinkle, roughening, skin laxity, paleness, telangiectasia, color changes, lentigo and speckles [1].
Chemical skin peeling treatment is an effective and reliable method employed in order to rule out and treat skin aging. It is generally applied to treat pigmentation disorders, acne scars, actinic damage and aging-related wrinkles as well as photo aging treatment in order to provide a healthier and younger facial appearance $[1,2]$.

Address for correspondence: Selda Yildirim, Dermatology Department, Atakent Acibadem Hospital, Acibadem Unıversity, Halkalı Merkez Mah. Turgut özel Bulvarı no: 16, 34307 Istanbul, Turkey, phone: +90 2124044232, e-mail: drselday@gmail.com Received: 16.03.2015, accepted: 5.05.2015. 
That the skin aging was reduced by the retinoid was firstly seen in female patients who were subject to acne treatment and it was reported that these patients had smoother skin and less wrinkles following that treatment. Compatible with these findings, the effects of this active substance upon the eradication of aging signs were commenced to be studied. In many controlled trials carried out for this purpose, it was observed that topically applied retinoic acid caused recuperation of crowded irregular pigmentation and thin lines in a pronounced way in the photo aged skin [1-3].

When examining the skin rejuvenation related studies, it is found out that chemical peeling treatment with trichloroacetic acid (TCA) has provided fairly good results. Despite being a very popular and commonly used chemical peeling agent, TCA requires a notable concern and appropriate use for successful results. On the other hand, peeling treatment with $0.1 \%$ retinoic acid is a cheap, efficient and feasible option but not as popular as TCA peeling. When reviewing the literature, no comparative studies between peeling treatment with $0.1 \%$ retinoic acid and TCA peeling were carried out for the facial rejuvenation.

\section{Aim}

The purpose of this study is to compare the efficiency, cosmetic results and possible adverse effects of the peeling treatment with $25 \%$ TCA and $0.1 \%$ retinoic acid for facial rejuvenation in the patients presenting with skin aging.

\section{Material and methods}

This study was conducted on 50 female patients in total reporting to the Okmeydani Training and Research Hospital Dermatology Department between January 2010 and August 2010 with complaints such as medium or advanced degree skin aging (Glagou 2-3).

Those patients who were treatment naïve, and could participate in the follow-up visits regularly, with 2-4 type Fitzpatrick skin, and who were between 30 and 60 years old were enrolled into this study. Patients participating in this study were subject to examination in order to make sure that they did not have any active bacterial, viral or fungal infection in the treatment region, and that they had not presented with inflammatory dermatosis such as psoriasis and atopic dermatitis, as well as significant collagen tissue disease, cardiovascular, pulmonary, renal or psychiatric disease history.

Patients using medications which might lead to photosensitization or patients with photosensitive disease, and having a tendency for a hypertrophic scar or keloid, who were undergoing the isotretinoin treatment for the last 6 months, who were recently operated in the facial region, who expected implausible results from this study, with Fitzpatrick 5-6 skin type and might not participate in the follow-up visits, as well as pregnant women and nursing mothers were excluded from this study.
Eligible patients were divided into 2 separate treatment groups. The first group patients were applied with TCA skin peeling with $25 \%$ concentration, which would cover all the facial region of the patient 4 times in total per month. The other group was subject to peeling treatment with $0.1 \%$ retinoic acid to be applied 5 nights of weekdays during the whole 4 months. The treatment options for the patients were determined in a randomized way (heads or tails method).

All the characteristics of the lesions of the patients were examined in detail prior to the treatment, and they were recorded in the patient follow-up form. In addition, the implementation sites were digitally photographed in the same environment, from the same angle during monthly follow-up visits before the treatment. All the patients were asked to reply to the 11 questions available in the Turkish Dermatology Life Quality Scale questionnaire about the social, emotional status, and daily activities as well as sexual life and symptoms of those patients during the last month [4].

All the patients were informed about the scope of the study thereby enabling them to read the patient information form prior to the treatment. Then, the signed consent forms were received.

Prior to the chemical peeling with TCA, the skin was washed with water and soap. Afterwards, gauze strips were submerged into the alcohol solution and the makeup stuff and oil were removed from the facial region. Hairs were away thanks to hair strip or bonnet. Eyes were protected with a wet gauze strip during the application. In order to prevent the acid accumulation in the periphery of eyes, the patient was supine, and the eyes were closed and the head was $45^{\circ}$ ahead. The medial and lateral canthus of the eyes, and the nasolabial folds, nostrils, lips commissure and the lips itself were applied with Vaseline in order to be protected from acid solution.

When the patient was sitting, the forehead, cheeks, nose, and jaws were applied with the $25 \%$ concentration TCA using a soft-end brush.

A ventilator was used in order to reduce the burn feeling. Following the application, following 1.5-2 min, after the frosting formation, the TCA solution on the facial region was neutralized with $10 \%$ sodium bicarbonate.

Following this procedure, the skin of the patient was washed with water. After this, solely an epithelial cream and sunlight protector cream were applied. The patients were informed in detail about being protected from the sun and using the sunlight protector cream. The peeling applications were carried out four times in total once per month.

The patients in the other group used $0.1 \%$ retinoic acid cream for 4 months by not diffusing to eyes and eyelids, and lips and nasal mucosa as a thin layer, and they applied every night of the weekday and waited till the morning. The patients were informed in detail about being protected from the sun and using the sunlight protector cream. Retinoic acid treatment was arranged as 
to be continued for 5 weekdays and to be paused for weekends, 2 days.

The evaluation was done by one responsible physician and 3 dermatologists in total. For this, all the observers comparatively evaluated by pointing the quartile scale prepared in advance, and the 4 separate regions i.e. the right cheek, left cheek, forehead and jaws with the signs as (0) no response, (1) minimal response, (2) partial response, (3) good response and (4) optimal response.

This evaluation was done without the knowledge of the patient treatment match by the observers except the responsible one. Computer software was designed and developed for this evaluation (Figure 1). Therefore, this evaluation would be stored using the software and the relevant documents would be easily retrieved thereby enabling a more reliable evaluation.

In addition to the efficiency of the treatment, this study was planned to evaluate the degree of burning, and irritation. Immediately following the chemical peeling treatment, the patients were asked to choose one of these four options, 5 - Likert scale (0-4): none (0), very mild (1), mild (2), severe (3) and very severe (4). All the patients were re-asked to reply to the 11 questions available in the Turkish Dermatology Life Quality Scale questionnaire about the social, emotional status, and daily activities as well as sexual life and symptoms of those patients during the last month [4].
Both these questionnaires were compared and it was planned to measure the change in the quality of life. In addition, patients' satisfaction with the treatment was compared by considering the pretreatment and post treatment, as well as final control analogue scales.

In addition to descriptive statistical methods (median, standard deviation, frequencies, average), a Student $t$ test was adopted in the comparisons of the quantitative data between the groups with normal parameters when the study data were evaluated.

\section{Statistical analysis}

On the other hand, Mann Whitney $U$ test was adopted for the inter group comparisons without normal distribution. Wilcoxon sign test was adopted in the group comparisons without normal distribution. For the compatibility among the evaluators, an intraclass correlation coefficient system was used. When comparing the qualitative data, $\chi^{2}$ test and Fisher's Exact $\chi^{2}$ test were adopted. The significance was evaluated as to be $p<0.05$.

\section{Results}

This study was implemented with 50 female patients in total with medium and advanced degree skin aging. The ages of the patients were among 30 and 57 and the mean age was $40.74 \pm 6.74$.
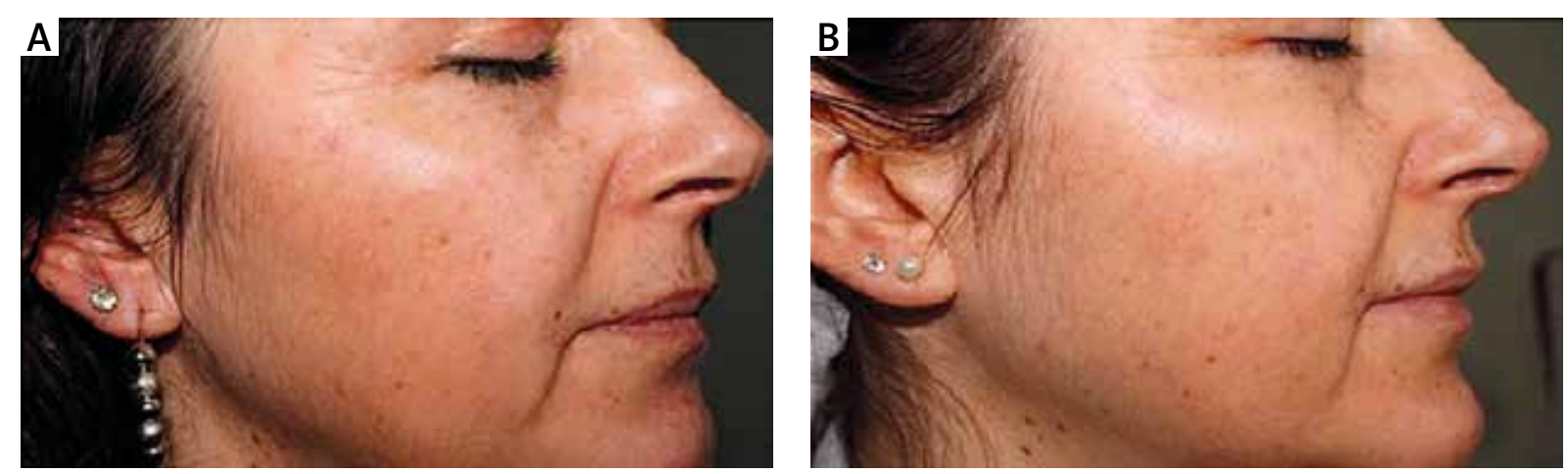

Figure 1. Before treatment (A) and follow-up (B) photos of a patient subjected to TCA treatment shown on the evaluation software interface

Table 1. Distribution of demographic information

\begin{tabular}{lcccc}
\hline Variable & & TCA $(n=24)$ & $\begin{array}{c}\text { Retinoic acid } \\
(n=26)\end{array}$ & $\begin{array}{c}\text { Total } \\
(n=50)\end{array}$ \\
\hline Age, mean \pm SD & & $42.58 \pm 6.70$ & $39.03 \pm 6.44$ & $40.74 \pm 6.74$ \\
\hline Sun effect, $n(\%)$ & None & $10(41.7)$ & $16(61.5)$ & $26(52.0)$ \\
\cline { 2 - 4 } & Yes & $14(58.3)$ & $10(38.5)$ & $24(48.0)$ \\
\hline Skin type, $n(\%)$ & Fitzpatrick 2 & $10(41.7)$ & $10(38.5)$ & $20(40.0)$ \\
\cline { 2 - 4 } & Fitzpatrick 3 & $12(50.0)$ & $15(57.7)$ & $27(54.0)$ \\
\hline
\end{tabular}


Twenty-four of the patients in this study were applied with 25\% TCA peeling treatment and the remaining 26 patients were applied with $0.1 \%$ retinoic acid cream treatment. No statistically significant difference $(p>0.05)$ was seen between the skin types and their duration of sunlight exposure and both groups were similar (Table 1).

At the end of the four applications, according to all the three observers, the healing rate in those patients applied with TCA chemical peeling was $33.3 \%$ while for retinoic acid cream treatment was $38.5 \%$. Compared to the pretreatment "average exchange levels" were not statistically significant considering all the levels observed in the post treatment process by these observers $(p>$ 0.05) (Table 2).

According to the three observers in the last followup visit in the third month following the treatment, the average healing rate in patients with chemical peeling method with TCA was $30.2 \%$ while $36.5 \%$ in the retinoic acid cream treatment group (Figure 2). In the last followup visit, according to the first and second observer, the healing rates in the retinoic acid cream treatment group were significantly higher than in the TCA group. However, according to the third observer, the healing rates in the retinoic acid cream treatment were higher than in the TCA group and this rate was not significant $(p>0.05)$ (Table 3).

Results of these three observers were assessed with an interclass correlation test and they were compatible with each other $(p<0.01)$.
Burning - irritation complaint was in all the visits detected to be more severe in the TCA group compared to the retinoic acid cream treatment group $(p<0.01)$ (Table 4$)$.

The frequency of TCA and retinoic acid cream treatment associated adverse effects was similar. No adverse effects were detected in the 34 patients out of 50 . Twenty-two percent of the patients $(n=11)$ presented with hyperpigmentation, in $78 \%(n=39)$ of the patients no such adverse effect was detected. No statistically significant difference $(p>0.05)$ between hyperpigmentation prevalence rates was seen in the groups. No scar formation was seen in any of the patients, and solely one patient was detected with hypopigmentation. In $8 \%$ of the patients $(n=4)$, irritation was seen in the skin, the remaining $92 \%$ did not demonstrate such an adverse effect (Table 5).

As a result of both applications, a pronounced decrease in the visual analogue patient satisfaction scores which show the patients' satisfaction with the treatment method was detected $(p<0.01)$ (Table 6).

Life quality scores of the patients with skin aging reduced as a result of both applications and a pronounced improvement in the quality of life $(p=0.001)$ (Table 7).

\section{Discussion}

Skin aging is a complex case including two different processes, intrinsic and extrinsic [5, 6]. Intrinsic aging is characterized by clinical, histological, and physiological changes in the skin and it is associated with the genetic

Table 2. Evaluation of mean changes after and before treatment

\begin{tabular}{|c|c|c|c|}
\hline \multirow[t]{2}{*}{ Variable } & TCA & Retinoic acid & \multirow[t]{2}{*}{$P$-value } \\
\hline & Mean \pm SD (median) & Mean \pm SD (median) & \\
\hline $1^{\text {st }}$ observer & $26.30 \pm 10.42(25)$ & $30.76 \pm 15.70(31.2)$ & 0.253 \\
\hline $2^{\text {nd }}$ observer & $34.11 \pm 8.23(34.37)$ & $39.42 \pm 13.55$ (37.5) & 0.121 \\
\hline $3^{\text {rd }}$ observer & $39.06 \pm 10.13(37.5)$ & $39.90 \pm 12.88(40.62)$ & 0.104 \\
\hline Mean & $32.55 \pm 8.21(33.3)$ & $37.58 \pm 12.76(38.5)$ & 0.152 \\
\hline
\end{tabular}
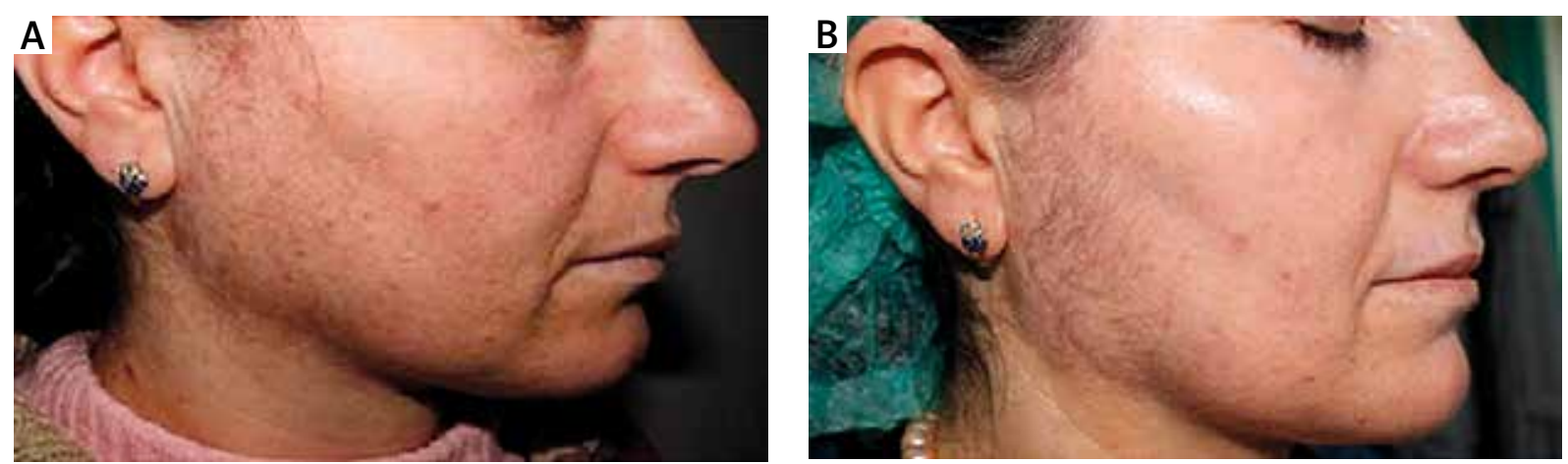

Figure 2. Before (A) and after (B) retinoic acid treatment photos of a patient 
Table 3. Evaluation of mean levels of control changes with respect to before treatment

\begin{tabular}{lccc}
\hline Variable & TCA & Retinoic Acid & \multirow{2}{*}{ P-value } \\
\cline { 2 - 3 } & Mean \pm SD (median) & Mean \pm SD (median) & \\
\hline $1^{\text {st }}$ observer & $22.13 \pm 12.08(25)$ & $29.81 \pm 14.17(31.25)$ & $0.042^{*}$ \\
\hline $2^{\text {nd }}$ observer & $29.42 \pm 9.83(28.12)$ & $35.81 \pm 11.53(37.5)$ & 0.423 \\
\hline $3^{\text {rd }}$ observer & $34.89 \pm 8.62(37.5)$ & $37.74 \pm 11.11(37.5)$ & 0.148 \\
\hline Mean & $29.34 \pm 9.75(30.2)$ & $34.93 \pm 11.07(36.5)$ & 0.085 \\
\hline
\end{tabular}

*Mann-Whitney $U$ test used $p<0.05$.

Table 4. Evaluation of burn - sting scores of groups

\begin{tabular}{|c|c|c|c|c|}
\hline \multirow[t]{2}{*}{ Burn - sting scores } & & \multirow{2}{*}{$\begin{array}{c}\text { TCA }(n=24) \\
n(\%)\end{array}$} & \multirow{2}{*}{$\begin{array}{c}\text { Retinoic acid }(n=26) \\
n(\%)\end{array}$} & \multirow[t]{2}{*}{$P$-value } \\
\hline & & & & \\
\hline \multirow[t]{3}{*}{ First follow-up } & Very mild & $2(8.3)$ & $15(57.7)$ & $0.001^{* *}$ \\
\hline & Severe & $13(54.2)$ & $11(42.3)$ & \\
\hline & Very severe & $9(37.5)$ & $0(0)$ & \\
\hline \multirow[t]{5}{*}{ Last follow-up } & None & $0(0)$ & $25(96.2)$ & $0.001^{\star *}$ \\
\hline & Very mild & $7(29.2)$ & $1(3.8)$ & \\
\hline & Mild & $13(52)$ & $0(0)$ & \\
\hline & Severe & $3(12.5)$ & $0(0)$ & \\
\hline & Very severe & $1(4.2)$ & $0(0)$ & \\
\hline \multicolumn{2}{|c|}{ - First follow-up - last follow-up } & 0.067 & $0.001^{\star \star}$ & \\
\hline
\end{tabular}

**Ki-Kare test used $p<0.01, \cdot$ Wilcoxon signed ranks test.

Table 5. Side effect occurrence

\begin{tabular}{|c|c|c|c|c|c|}
\hline \multirow[t]{2}{*}{ Side effect } & & TCA & Retinoic acid & Total & $P$-value \\
\hline & & $n(\%)$ & $n(\%)$ & $n(\%)$ & \\
\hline \multirow[t]{2}{*}{ Hypopigmentation } & Yes & $1(4.2)$ & $0(0)$ & $1(2)$ & - \\
\hline & None & $23(95.8)$ & $26(100)$ & $49(98)$ & \\
\hline \multirow[t]{2}{*}{ Hyperpigmentation } & Yes & $5(20.8)$ & $6(23.1)$ & $11(22.0)$ & 0.848 \\
\hline & None & $19(79.2)$ & $20(76.9)$ & $39(78.0)$ & \\
\hline \multirow[t]{2}{*}{ Scars } & Yes & - & - & - & - \\
\hline & None & $24(100)$ & $26(100)$ & $51(100)$ & \\
\hline \multirow[t]{2}{*}{ Skin irritation } & Yes & $0(0)$ & $4(15.4)$ & $4(8.0)$ & 0.111 \\
\hline & None & $24(100)$ & $22(84.6)$ & $46(92.0)$ & \\
\hline
\end{tabular}

Table 6. Comparison of groups with respect to VAS measurements

\begin{tabular}{|c|c|c|c|}
\hline \multirow[t]{2}{*}{ Variable } & TCA $(n=25)$ & Retinoic acid $(n=26)$ & \multirow[t]{2}{*}{$P$-value } \\
\hline & Mean \pm SD (median) & Mean \pm SD (median) & \\
\hline $1^{\text {st }}$ follow-up & $7.60 \pm 1.36(8)$ & $7.0 \pm 1.61(7)$ & 0.163 \\
\hline $3^{\text {rd }}$ follow-up & $3.73 \pm 1.43(4)$ & $3.12 \pm 1.57$ (3) & 0.112 \\
\hline${ }^{++} 1^{\text {st }}$ follow-up $-3^{\text {rd }}$ follow-up & $0.001^{\star \star}$ & $0.001^{\star *}$ & \\
\hline
\end{tabular}

Mann-Whitney $U$ test used: ${ }^{++}$Wilcoxon signed ranks test, ${ }^{* *} p<0.01$. 
Table 7. Evaluation of groups with respect to quality of life in dermatology scores

\begin{tabular}{lccc}
\hline Quality of life in dermatology & TCA $(n=24)$ & Retinoic acid $(n=26)$ & \multirow{2}{*}{$P$-value } \\
\cline { 2 - 3 } & Mean \pm SD (median) & Mean \pm SD (median) & \\
\hline Before treatment & $21.20 \pm 10.13(24)$ & $19.73 \pm 8.35(21)$ & 0.478 \\
\hline After treatment & $11.78 \pm 8.74(11.0)$ & $10.12 \pm 5.69(8)$ & 0.528 \\
\hline${ }^{+}$Before treatment - after treatment & $0.001^{\star \star}$ & $0.001^{\star \star}$ & \\
\hline
\end{tabular}

Mann-Whitney $U$ test used: ${ }^{++}$Wilcoxon signed ranks test, ${ }^{* *} p<0.01$.

structure of the patient [7]. Extrinsic skin aging on the other hand is associated with smoking, excessive alcohol use, insufficient nutrition and negative environment factors such as sunlight [8]. As the sun exposure is usually the most significant environmental factor, it is frequently called "photo aging".

In addition to its psychological, social relations and work life effects, skin aging is of great importance considering its inducing effect on precancerous and cancerous skin Ca development and it must necessarily be treated [9].

Chemical skin peeling method decreases the photo aging with dermal volume increase and rearrangement in the dermal structural elements. In a study, Butler et al. irradiated the photo aging rat groups with UVB during 14 weeks and applied chemical peeling treatment with glycolic acid 50\%, TCA 30\%, TCA 50\% and phenol and all the rats were exposed to punch biopsy on day 14, 28 and 60 .

Compatible with this, when compared to the control group, a significant increase in the dermal thickness and glycosaminoglycan amount in the TCA and phenol groups and rearrangement in the dermal collagen and elastic fibrils were observed [10].

Trichloroacetic acid skin peeling treatment has been used for a long time in the treatment of actinic keratosis and other dermatoses. The first use in the treatment of skin aging was defined in the studies of Ayres [11, 12]. It has been considered as a gold standard for a long time, which does not cause systemic toxicity, is stable and which does not require neutralization in the application [13].

Trichloroacetic acid in the facial rejuvenation treatment has been used with various concentrations and the necrotic depth due to the increase in the concentrations used is also increased. In a study by Brodland et al., TCA solutions with $20 \%, 35 \%, 50 \%$ and $80 \%$ concentrations were applied to the hairless rats and in the histopathological examinations it was also determined that the skin necrosis and therefore the rejuvenation rate increased [14].

The most frequent complication following the chemical peeling with TCA is hyperpigmentation associated with early sunlight exposure or non-protection from sunlight [15].

In our study, it was detected that two of the $5 \mathrm{pa}$ tients undergoing TCA did not regularly use sunlight protectors and the remaining three patients presented with hyperpigmentation due to early sunlight exposure.
The less frequent adverse effect following the peeling treatment with TCA is persistent erythema. It generally occurs with some symptoms such as itching, burning and irritation as well as deterioration in the skin tissue [16].

This case leading to scar formation should be treated with topical potent corticosteroids [17]. In our study, only 1 patient presented with persistent erythema following the third peeling application. This patient was treated with mometasone furoate for 2 weeks. After 2 weeks, erythema, itching and burning complaints of the patient were eradicated.

Systemic and topical retinoid have intensively been used particularly in the acne treatment in the dermatology departments. The first anecdotal data related to the fact that skin aging was healed with retinoid were acquired from the female patients undergoing the acne treatment and they reported that they had smoother skin and less speckles after this treatment. These observations were also supported with the clinical studies [18-20].

The first animal experiment on the effects of topical retinoid in the treatment of photo aging was implemented by Kligman et al. in 1896 [21]. For this, the dermal damage was done with FS20 irradiation lamp on the hairless rats during 10 weeks and the rats were then treated with topical retinoic acid of different concentrations after 5-10 weeks.

When the skin of these rats was evaluated by using irradiation and electron microscopic techniques, compared to non-treated control group rats, the sub epidermal repair zone was pronouncedly large. In addition, it was determined that dermal vascularity of the rats treated increased and that fibroblasts were morphologically activated [22].

The first study associated with the retinoid effects of the photo aging on the human skin was done by Kligman et al. in 1986. In this study, patients with photo aged skin were subject to $0.05 \%$ tretinoin cream treatment on their face and forearms, and a clinical, histological, ultrastructural and physiological reduction was determined in the regions compared to sites treated with placebo [23].

The studies supported the fact that topical retinoid was effective in the treatment of photo aging as well as its role in prevention. Fisher et al. reported that the tretinoin application with UVB caused inhibition of inductions of the MMPs [24]. In another study, Fisher et al. 
showed that the early treatment of the skin with all-trans retinoic acid inhibited the loss of procollagen synthesis developed due to UV contact [25]. Therefore, if used in an appropriate way, it provides an effective protection against the photo aging with topical retinoid [26].

The most frequent adverse effect related to the use of topical retinoid use is the skin irritation characterized by peeling and redness. The important aspect is to differentiate the skin irritation from the effects of retinoid leading to photo aging and to tell the patient that retinoid was effective in the treatment despite not developing desquamation.

In our study, although no pronounced desquamation was observed in 3 patients during the study, at the end of the study, the effects of photo aging of these cases were significantly recovered. Another adverse effect related to topical retinoid was erythema occurring with independent mechanisms, this was developed in solely four patients in our study but it was spontaneously recovered.

According to all the three observers, after the four months' treatment as a result of image evaluation, the recuperation rate with acid treatment was detected to be higher than in the cases applied with TCA chemical peeling method.

However, this was not statistically significant. All the observers evaluating the last control images compared to the initial images reported that photo aging results were more decreased compared to those patients who applied TCA. According to two observers, this recuperation was more significant in the retinoic acid group compared to the TCA group and we consider that this significance will increase in larger studies.

Recently, various invasive and non-invasive methods have been adopted in order to prevent or rule out the effects of skin aging. One of these methods, skin peeling with TCA has been used successfully used in the treatment of photo aging. Trichloroacetic acid, despite being a popular and commonly used chemical peeling agent, must be used in an appropriate and careful way. On the other hand, the photo aging treatment with retinoic acid is cheaper compared to TCA, is feasible for the patient use, and as effective and reliable as TCA. The accurate and correct information of the patient by the physician is of great importance. In both treatments, photo aged skin will be recovered with the best results if the rules and relevant procedures are complied with as well.

\section{Conflict of interest}

The authors declare no conflict of interest.

\section{References}

1. Moyal D, Fourtanier A. Acute and chronic effects of UV on skin. What are they and how to study them? In: Photoaging. Rigel DS, Weiss RA (eds.). Marcel Dekker, Inc. New York 2004; 15-32.
2. Aybey B, Ergenekon G. The use of chemical peeling alpha hydroxi acid, new generation hydroxi acid and complex polihydroxi acid in dermatology. Hipokrat Dergisi 2004; 13: 115-21.

3. Koçyiğit P, Bostancı S. Chemical peeling. T Klin J Dermatol 1997; 7: 143-50.

4. Gurel MS, Yanik M, Simsek Z, Kati M, Karaman A. Quality of life instrument for Turkish people with skin diseases. Int J Dermatol 2005; 44: 933-8.

5. Uitto J, Bernstein EF. Molecular mechanisms of cutaneous aging: connective tissue alterations in the dermis. J Investig Dermatol Symp Proc 1998; 3: 41-4.

6. Watson RE, Griffiths CE. Pathogenic aspects of cutaneous photoaging. J Cosmet Dermatol 2005; 4: 230-6.

7. Roenigk HH Jr. Treatment of the aging face. Dermatol Clin 1995; 13: 245-61.

8. Chung JH, Hanft VN, Kang S. Aging and photoaging. J Am Acad Dermatol 2003; 49: 690-7.

9. Lawrence N. New and emerging treatments for photoaging. Dermatol Clin 2000; 18: 99-112.

10. Butler PE, Gonzalez S, Randolph MA, et al. Quantitative and qualitative effects of chemical peeling on photo-aged skin: an experimental study. Plast Reconstr Surg 2001; 107: 222-8.

11. Roenigk RK, Brodland DG. A primer of facial chemical peel. Dermatol Clin 1993; 11: 349-59.

12. Ayres S 3rd. Superficial chemosurgery in treating aging skin. Arch Dermatol 1962; 85: 385-93.

13. Ghersetich I, Teofoll P. Chemical peeling: how, when, why? J Eur Acad Dermatol Venerol 1997; 8: 1-11.

14. Brodland DG, Cullimore KC, Roenigk RK. Depths of chemexfoliation induced by various concentrations and application techniques of trichloroacetic acid in a porcine model. J Dermatol Surg Oncol 1989; 15: 967-71.

15. Mendelsohn JE. Update on chemical peels. Otolaryngol Clin North Am 2002; 35: 55-72.

16. Maloney BP, Millman B, Monheit G, McCollough EG. The etiology of prolonged erythema after chemical peel. Dermatol Surg 1998; 24: 337-41.

17. Nguyen TH, Rooney JA. Trichloroacetic acid peels. Dermatol Ther 2000; 13: 173-82.

18. Goldfarb MT, Ellis CN, Voorhees JJ. Topical tretinoin: its use in daily practice to reverse photoageing. Br I Dermatol 1990; 122 (Suppl 35): 87-91.

19. Gilchrest BA. Treatment of photodamage with topical tretinoin: an overview. J Am Acad Dermatol 1997; 36: S27-36.

20. Griffiths CE. Assessment of topical retinoids for the treatment of Far-East Asian skin. J Am Acad Dermatol 1998; 39: S104-7.

21. Varani J, Fisher GJ, Kang S, Voorhees JJ. Molecular mechanisms of intrinsic skin aging and retinoid-induced repair and reversal. J Investig Dermatol Symp Proc 1998; 3: 57-60.

22. Kligman LH. Effects of all trans-retinoic acid on the dermis of hairless mice. J Am Acad Dermatol 1986; 15: 779-85.

23. Kligman AM, Grove GL, Hirose R, Leyden JJ. Topical tretinoin for photoaged skin. J Am Acad Dermatol 1986; 15: 836-59.

24. Fisher GJ, Datta SC, Talwar HS, et al. Molecular basis of suninduced premature skin ageing and retinoid antagonism. Nature 1996; 379: 335-9.

25. Fisher GJ, Datta S, Wang Z. c-Jun-dependent inhibition of cutaneous procollagen transcription following ultraviolet irradiation is reversed by all-trans retinoic acid. J Clin Invest 2000; 106: 663-70.

26. Fisher GJ, Talwar HS, Lin J. Molecular mechanisms of photoaging in human skin in vivo and their prevention by all-trans retinoic acid. Photochem Photobiol 1999; 69: 154-7. 